\title{
Microemulsion Polymerization of Butyl Acrylate in the Presence of Polymeric Hydrophobes
}

\author{
Ignac CAPEK \\ Polymer Institute, Slovak Academy of Sciences, Dúbravská cesta 9, 84236 Bratislava, Slovak Republic
}

(Received January 13, 2000)

\begin{abstract}
Microemulsion polymerization of butyl acrylate initiated by a water-soluble ammonium peroxodisulfate initiator in the presence of polymeric hydrophobe was investigated. The polymerization rate (without polymer) vs. conversion curve shows two nonconstant rate intervals. The addition of polymeric hydrophobe promotes increase of maximum rate of polymerization, appearance of a rate shoulder at ca. 50--70\% conversion and formation of polymer particles with bimodal size distribution. The maximum rate of polymerization increases with initiator concentration, particularly so with predissolved polymer. The enhanced polymerization rate with predissolved polymer is attributed to preservation of monomer droplets and larger number of latex particles. This is observed in runs with a higher weight ratio of monomer to emulsifier. The synergetic effect of predissolved polymer is also attributed to the incompatibility of polymer with (co)emusifier (BA and undissociated emulsifier), which may increase the fraction of micellar emulsifier. The non-stationary rate of polymerization is discussed in terms of continuous particle nucleation, decrease of monomer at the reaction loci, increase in weight ratio emulsifier/monomer or chain transfer to emulsifier and preservation of monomer droplets.
\end{abstract}

KEY WORDS Microemulsion / Butyl Acrylate / Polymeric Hydrophobe / Rate of Polymerization / Latex Particles /

The principle behind the formation of transparent or semitransparent microemulsions (with a droplet size 10 $-60 \mathrm{~nm})$ is very low interfacial tension caused by decreased dissociation of emulsifier and penetration of coemulsifier into the droplet surface layer. Coemulsifier is responsible for the enormous increase in the droplet surface area (or droplet number). Three- or four-component mixtures containing water, monomer, emulsifier and coemulsifier form not only kinetically stable emulsions but also thermodynamically stable microemulsions. Fourcomponent mixtures containing water, monomer, emulsifier and hydrophobe form mostly kinetically stable miniemulsions. The second class of fine emulsions so called miniemulsions (droplet size, $100-300 \mathrm{~nm}$ ) are relatively stable submicron dispersions of monomer in water prepared by shearing a system containing monomer (oil), water, emulsifier and hydrophobe. The principle behind the making of stable miniemulsions is the introduction of a hydrophobic compound into the monomer phase to retard diffusion of monomer out of the monomer droplets. In both systems, the principal locus of particle nucleation is the emulsified monomer droplet. In the former case the dependence of the rate us. conversion is described by a curve with a maximum at $c a .20-$ $40 \%$ conversion and nucleation proceeds to the final conversion. ${ }^{1}$ In the latter case particle nucleation is somewhat shortened up to $c a .30-50 \%$ and the dependence of the rate $v$ s. conversion is described by a curve with two maxima (four rate intervals). ${ }^{2}$

Micro- and miniemulsions exhibit large interface between oil and water. There was no apparent constant rate period in thermally initiated micro- and miniemulsion polymerizations. ${ }^{1-6}$ Ugelstad et al. ${ }^{6}$ clearly demonstrated that monomer droplets effectively compete with monomer-swollen micelles for capture of free radicals generated in the aqueous phase when total surface area of the droplets became large enough. In the conventional emulsion polymerization, the principle locus of particle nucleation is the aqueous phase or monomer swollen micelles depending on the degree of water solubility of monomer(s) and amount of emulsifier. Monomer droplets are considered to act as monomer reservoir supplying monomer to the growing polymer particles. In the micro- or miniemulsion polymerization, the small size of monomer droplets (micro- or minidroplets) enables them to become the principle locus of particle nucleation.

In the microemulsion polymerization of unsaturated monomers (styrene, alkyl (metha) acrylates, etc.), the initial generation of polymer paralleled strong increase in turbidity. ${ }^{1,7}$ The accumulation of polymer was supposed to decrease the colloidal stability of fine emulsion (thermodynamically stable microemulsion changed to kinetically stable one) which promotes the formation of large polymer particles. To model this behavior the effect of predissolved polymer on the polymerization process was investigated. The presence of polymer (hydrophobe) in the monomer droplets is supposed to suppress the diffusion of monomer out of the monomer droplets and preserve the monomer droplets for a longer period of polymerization time. Thus, the main item of this work was to follow the effects of polymeric hydrophobe such as PSt, PMMA, etc. on the kinetics of microemulsion polymerization of butyl acrylate.

\section{EXPERIMENTAL}

\section{Materials}

Commercially available butyl acrylate(BA) was purified by usual methods. ${ }^{7,8}$ The analytical-grade initiator ammonium peroxodisulfate(APS) was used as supplied (Fluka). Polystyrene(PSt), poly(methyl methacrylate) (PMMA), poly(butyl acrylate)(PBA), and polyvinylpyridone(PVP) were used as supplied (Fluka). The emulsifier used was reagent-grade sodium dodecyl sulfate 
(SDS) (Fluka). Twice-distilled water was used as the polymerization medium.

\section{Recipe and Procedures}

Batch polymerizations were run at $60^{\circ} \mathrm{C}$. In all runs the recipe comprises $100 \mathrm{~g}$ water, $20 \mathrm{~g}$ SDS, and $0.031 \mathrm{~g}$ $\mathrm{NaHCO}_{3}$. Amounts of APS, BA and polymer varied as shown later. The polymerization technique, conversion determination (dilatometric and gravimetric techniques) and estimation of polymerization rate were the same as described earlier., ${ }^{4,9}$ Measurement of average (monomer-swollen) particle size (a static and dynamic light scattering-LS) was the same as described earlier. ${ }^{9,10}$ Apparent particle size distribution, APSD, was estimated as described by Schork et al. ${ }^{11}$ The coefficient of variation and polydispersity index were related according to Hunter's approach. ${ }^{12}$ Limiting viscosity numbers $[\eta]$ were determined with Ubbelohde viscometer in acetone at $30^{\circ} \mathrm{C}$ and used to estimate the viscosity-average molecular weights. ${ }^{7,9}$ The surface tension of polymer latexes was measured by the stalagmometric method at $20^{\circ} \mathrm{C} \cdot{ }^{13,14}$

\section{RESULTS AND DISCUSSION}

\section{Rate of Polymerization}

Variation in rate of fine emulsion polymerization $\left(R_{\mathrm{p}}\right)$ of BA with conversion and additive (PSt, PMMA, PVP) type is summarized in Tables I-III and in Figure 1. The polymerization rate $v s$. conversion curves show two distinct non-stationary rate intervals (runs 1-4, Table I, without polymer). The addition of polymeric hydrophobe (PSt) promotes the appearance of a rate shoulder at $c a$. $50-70 \%$ conversion (runs $5-8$, Table I). In runs $1-4$, the polymerization rate increases to a maximum ( $\mathrm{ca}$. at $30 \%$ ) followed by a final decrease. Similar behavior is observed in runs $5-8$, except for high conversion with the appearance of a rate shoulder. There is no significant constant rate interval, as a result of variation of particle and monomer concentration with conversion. The first abrupt increase in the rate of polymerization is attributed to intensive particle nucleation. ${ }^{1,7}$ The number of polymer particles $\left(N_{\mathrm{p}}\right)$ increased from $3 \times 10^{17} \mathrm{dm}^{-3}$ to $9 \times 10^{17} \mathrm{dm}^{-3}$ with conversion from $20 \%$ to $90 \%$, respectively (Table I, run 1). A similar trend was observed with PSt (run 5) but increase of $N_{\mathrm{p}}$ with conversion was much more pronounced, that is, $N_{\mathrm{p}}$ increased from $5 \times 10^{17}$ $\mathrm{dm}^{-3}$ to $13 \times 10^{18} \mathrm{dm}^{-3}$ with conversion from $20 \%$ to $90 \%$. In the microemulsion polymerization of BA or styrene, the monomer saturation conditions are operative below $c a$. $20 \%$ conversion while the monomer-starved conditions are operative above ca. $30 \%$ conversion. ${ }^{1,9}$ The monomer partitions between the (active and inactive) polymer particles and mixed micelles (interfacial layer saturated with monomer and monomer core does not appear). The decrease in rate with increasing conversion was attributed to decrease of monomer concentration at reaction loci due to increased dilution of monomer by emulsifier (hydrophobic chains of emulsifier) and polymer, and increased radical chain transfer to emulsifier.

Synergism in growth appeared in runs $5-9$ with PSt where both very small and large polymer particles ap- peared in the final polymer latex (Table I). Similar synergetic effect of predissolved PSt was found in the miniemulsion polymerization of styrene stabilized by SDS/ CA. ${ }^{15}$ The rate enhancement was attributed to preservation of the monomer droplet number by the presence of polymer in each monomer droplet. Polymer acts to preserve droplets for longer periods during polymerization compared to conventional fine emulsions. However, this effect was not observed in the absence of coemulsifier CA (cetyl alcohol). It was assumed that incompatibility between PSt and coemulsifier (alkyl alcohol) leads to preferential accumulation of coemulsifier at the droplet surface and increase of total surface area. ${ }^{16}$ Similar behavior (prefential accumulation of emulsifier at the droplet surface) can be expected for ionic emulsifier and PSt. Indeed, the fraction of SDS was detected in the styrene monomer core by Lin et al. ${ }^{17}$ The authors ${ }^{17}$ estimated that $c a .6 \%$ of the initially added SDS molecules is adsorbed on the monomer droplets in emulsion at $c a$. CMC and $5 \%$ of the SDS molecules absorbed inside the monomer droplets. The double emulsion is further evidence that ionic emulsifier penetrates the monomer droplet. ${ }^{18}$ The very high weight ratio emulsifier/water in the microemulsion ensures that the degree of dissociation of SDS is strongly depressed.,19 The counterions are strongly associated with amphiphiles, essentially creating a neutral but highly polar molecule. The ion pairs exist molecularly dispersed in equilibrium with associated emulsifier molecules. The principle behind the making of stable microemulsions lies in the decrease of micelle ionization or electrostatic repulsion between emulsifier molecules in the presence of a large amount of electrolyte (ionic emulsifier). Under these circumstances, the higher fraction undissociated emulsifier increases in microdroplets where it is molecularly dispersed or forms inverse micelles. This decreases the initial micellar fraction of emulsifier for particle nucleation and particle stabilization. The released emulsifier from the droplet during polymerization increases the colloidal stability of growing latex particles and continuous particle nucletion. (This may be one reason why the microemulsion kinetic models fail at high conversion ${ }^{20}$ ). The predissolved polymer (PSt and PMMA), however, is incompatible with undissociated emulsifier and coemulsifier (BA) and favors the accumulation of both (BA is bad solvent for PSt) at the droplet surface. The surface active BA accumulates close to the droplet surface ${ }^{9}$ (decreasing the surface tension of the reaction mixture ${ }^{21}$ ). This is not the case with PBA and PVP where polymer is well soluble in BA and probably more compatible with undissociated emulsifier. The emulsifier dissolved in the monomer phase decreases both the rate of polymerization and molecular weight. ${ }^{22}$ Tables I and II indicate that the synergetic effect of predissolved polymer is higher for lower molecular weight hydrophobes (PSt, PMMA, and PBA) and hydrophobe with higher polarity.

Variation of the polymerization rate $\left(R_{\mathrm{p}}\right)$ with increasing conversion can also be discussed in tems of particle number. In the beginning of polymerization (monomersaturated condition), $R_{\mathrm{p}}$ increases with particle number. The increase in $R_{\mathrm{p}}$ is more pronounced with predissolved PSt compared to that without polymer. After ca. 20$30 \%$ (monomer-starved condition) the decrease in $R_{\mathrm{p}}$ is 
Table I. Kinetics, molecular weights and colloidal parameters in the radical polymerization of BA in fine emulsions ${ }^{\text {a }}$

\begin{tabular}{|c|c|c|c|c|c|c|c|c|c|}
\hline \multirow{2}{*}{ \# } & \multirow{2}{*}{$\frac{[\mathrm{APS}] 10^{3}}{\mathrm{~mol} \mathrm{dm}^{-3}}$} & \multirow{2}{*}{ 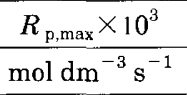 } & \multirow{2}{*}{$\frac{\text { Conv. }_{\text {max }}}{\%}$} & \multirow{2}{*}{$\frac{\text { Conv.f }_{\text {f }}}{\%}$} & \multirow{2}{*}{$\frac{D}{\mathrm{~nm}}$} & \multirow{2}{*}{$\frac{N_{\mathrm{p}} \times 10^{-18}}{\mathrm{dm}^{3}}$} & \multirow{2}{*}{ APSD } & \multirow{2}{*}{$M_{\mathrm{v}} \times 10^{6}$} & \multirow{2}{*}{$\frac{\gamma}{\mathrm{mN} \mathrm{m}^{-1}}$} \\
\hline & & & & & & & & & \\
\hline 1 & $1.0^{\mathrm{b}}$ & 4.5 & 28 & 89 & $69^{\mathrm{e}}$ & $0.94^{\mathrm{e}}$ & $1.1^{\mathrm{e}}$ & 2.7 & 43 \\
\hline 2 & $2.5^{\mathrm{b}}$ & 6.2 & 28 & 90 & $63^{\mathrm{e}}$ & $1.25^{\mathrm{e}}$ & $1.05^{\mathrm{e}}$ & 2.5 & \\
\hline 3 & $5.0^{\mathrm{b}}$ & 10 & 30 & 95 & $58^{\mathrm{e}}$ & $1.7^{\mathrm{e}}$ & $1.2^{\mathrm{e}}$ & & \\
\hline 4 & $10^{b}$ & 16 & 35 & 99 & $54^{e}$ & $2.2^{\mathrm{e}}$ & $1.2^{\mathrm{e}}$ & 1.7 & 38 \\
\hline 5 & $1.0^{\mathrm{c}}$ & 4.6 & 27 & 92 & $\begin{array}{c}62(98 \text { vol. } \%)^{\mathrm{e}} \\
560^{\mathrm{f}}\end{array}$ & $1.3^{\mathrm{e}} 36^{\mathrm{d}, f}$ & $1.4^{\mathrm{e}} 1.7^{\mathrm{f}}$ & 2.3 & 35 \\
\hline 6 & $2.5^{\mathrm{c}}$ & 10 & 27 & 92 & $50(80)^{\mathrm{e}} 400^{\mathrm{f}}$ & $2.0^{\mathrm{e}} \quad 0.001^{\mathrm{f}}$ & $1.5^{\mathrm{e}} 1.9^{\mathrm{f}}$ & 2.0 & \\
\hline 7 & $5.0^{\mathrm{c}}$ & 14.6 & 30 & 93 & $32(75)^{\mathrm{e}} 200^{f}$ & $0.01^{f}$ & $1.4^{\mathrm{e}} 1.4^{\mathrm{f}}$ & & \\
\hline 8 & $10^{\mathrm{c}}$ & 15.8 & 30 & 95 & $22(66)^{\mathrm{e}} \quad 62^{\mathrm{f}}$ & $4.7^{f}$ & $1.4^{\mathrm{e}} 1.3^{\mathrm{f}}$ & 1.8 & 30 \\
\hline
\end{tabular}

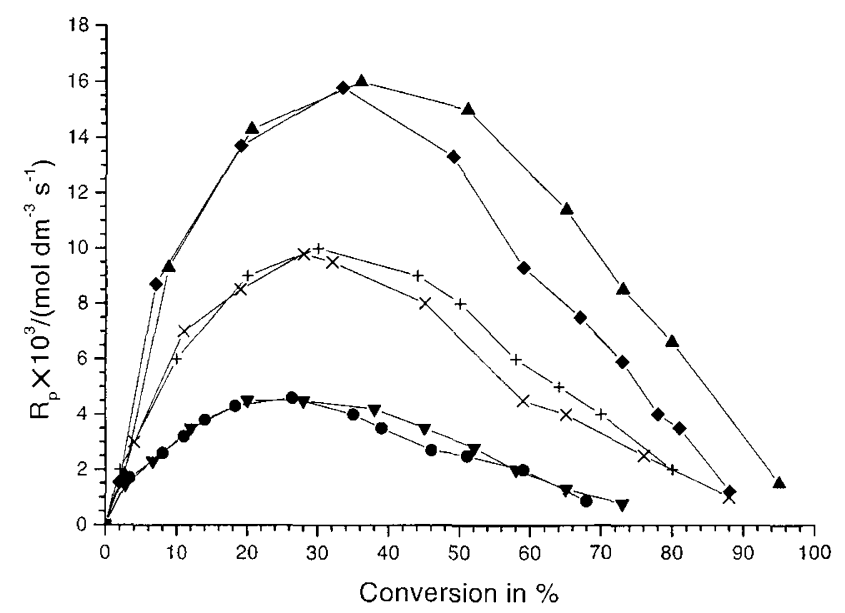

Figure 1. Variation of the polymerization rate in the microemulsion polymerization of $\mathrm{BA}$ with conversion and concentrations of APS and PSt $\left(M_{w}=1.8 \times 10^{6}\right)$. Recipe, $c f$. Experimental. [BA] $=$ $1.56 \mathrm{~mol} \mathrm{dm}^{-3}$, [APS] $/ \mathrm{mol} \mathrm{dm}^{-3}: 10(\Delta)$, without PSt), $10(\boldsymbol{B}$, with PSt), 5 ( + , without PSt), 2.5 ( $\times$, with PSt), 1.0 ( $\nabla$, without PSt), 1.0 (O, with PSt).

more pronounced in runs with higher number of particles (with predissolved PSt: the runs 5-8). Chain transfer to emulsifier decreases the rate of polymerization and the decrease is proportional to conversion. This results from increasing weight ratio emulsifier/monomer with conversion. The hydrophobic PSt accumulates in the monomer droplets (the highly-monomer swollen particles) with conversion and, thereby, depresses more the monomer droplet degradation ${ }^{11}$ (monomer concentration at the reaction loci) at high conversion (above $c a$. $30 \%)$.

The rate of polymerization, however, slightly decreased by the addition of hydrophobe (compare runs 4 and 8 , Table I). In run 8 the very fine polymer dispersion appeared. Similar behavior was observed in runs with a larger weight ratio emulsifier/monomer (Table III, runs 19-21). Under such circumstances, the monomerstarved condition governs the polymerization, that is, the surface active monomer is mainly located in the interfacial layer (mixed micelles) and, thereby, polymeri- zation is slower.

The slight increase in polymerization rate at high conversion can be attributed to the gel effect that increases polymer fraction and growing radicals in the latex particles. The depressed rate of termination inside the particle favors the accumulation of entangled radicals in polymer particles and increases the rate of polymerization. This is very similar to that reported by El-Aasser et al. ${ }^{23}$ and Shork et $a l^{11}$ for classical miniemulsion polymerization of styrene and MMA in the presence of low and high molecular weight hydrophobe such as hexadecane (HD), PMMA, PSt, etc. and homogenized by a uniform shear device. The second rate maximum, however, was not obtained in the microemulsion polymerization of alkyl (meth)acrylates or styrene performed at elevated temperatures where the dependence of the rate of polymerization vs. conversion was described by a curve with a single maximum at $20 \%-40 \%$ conversion. ${ }^{3}$ In microemulsion polymerization, the gel effect is supposed to be operative at low conversion; that is, it contributes to the maximum rate at $20 \%-40 \%$ conversion. In the classical miniemulsion polymerization using an effective hydrophobe (HD) the second rate maximum is more pronounced than the first rate maximum. ${ }^{24}$ In the presence of polymer (a poor hydrophobe), the second maximum is not distinct and appears as a rate shoulder. ${ }^{24}$

The relationship $R_{\mathrm{p}} v s$. [APS] ${ }^{x}$ is used to estimate the mechanism of radical termination and particle nucleation. The effect of APS concentration on the polymerization behavior is shown in Table I. Here, the reaction or$\operatorname{der} x$ was found to vary as follows:

\subsection{5 (the runs $1-4$, system $A)<0.73$ (the runs $5-7$, system B)}

The reaction order $x$ for both systems is larger than that (0.4) for the classical emulsion model. ${ }^{25}$ The number of polymer particles strongly increases with APS concentration and the increase is more pronounced for the system with PSt. The droplet preservation increases the rate of particle formation. In the miniemulsion polymerization, this behavior was attributed to the barrier for entering radicals into the polymer containing minidroplets. The increased rate of particle formation is also connected with increased release of emulsifier from the nu- 
Table II. Kinetics, molecular weights and colloidal parameters in the radical polymerization of BA in fine emulsions ${ }^{\mathrm{a}}$

\begin{tabular}{|c|c|c|c|c|c|c|c|c|c|c|}
\hline \multirow{2}{*}{ \# } & \multirow{2}{*}{ Polymer } & \multirow{2}{*}{$M_{w} \times 10^{-4}$} & \multirow{2}{*}{$\frac{R_{\mathrm{p}, \max } \times 10^{3}}{\mathrm{~mol} \mathrm{dm}^{-3} \mathrm{~s}^{-1}}$} & \multirow{2}{*}{$\frac{\text { Conv }_{\text {max }}}{\%}$} & \multirow{2}{*}{$\frac{\text { Conv.f }_{\text {f }}}{\%}$} & \multirow{2}{*}{$\begin{array}{c}D \\
\mathrm{~nm} \\
\end{array}$} & \multirow{2}{*}{$\frac{N_{\mathrm{p}} \times 10^{-18}}{\mathrm{dm}^{-3}}$} & \multirow{2}{*}{ APSD } & \multirow{2}{*}{$M_{\mathrm{v}} \times 10^{6}$} & \multirow{2}{*}{$\frac{\gamma}{\mathrm{mN} \mathrm{m}^{-}}$} \\
\hline & & & & & & & & & & \\
\hline 5 & Pst & 180 & 4.6 & 27 & 92 & $62(98)^{\mathrm{c}} 560^{\mathrm{d}}$ & $1.3^{\mathrm{c}} \quad 36^{\mathrm{b}, \mathrm{d}}$ & $1.4^{\mathrm{c}} \quad 1.7^{\mathrm{d}}$ & 2.3 & 35 \\
\hline 9 & Pst & 18 & 4.8 & 26 & 90 & $45(80)^{\mathrm{c}} 120^{\mathrm{d}}$ & $2.9^{\mathrm{c}} \quad 0.035^{\mathrm{d}}$ & & 2.4 & 32 \\
\hline 10 & PMMA & 180 & 4.6 & 28 & 90 & $69(98)^{c} 600^{d}$ & $0.95^{\mathrm{c}} 30^{\mathrm{b}, \mathrm{d}}$ & & 2.0 & 33 \\
\hline 11 & PMMA & 16 & 5.2 & 27 & 89 & $62(95)^{\mathrm{c}} 500^{\mathrm{d}}$ & $1.3^{\mathrm{c}} 123^{\mathrm{b}, \mathrm{d}}$ & $1.35^{\mathrm{c}}$ & 2.4 & 32 \\
\hline 12 & PMMA & 1.9 & 4.2 & 22 & 92 & $41(86)^{\mathrm{c}} \quad 94^{\mathrm{d}}$ & $4.0^{\mathrm{c}} \quad 0.054^{\mathrm{d}}$ & & 2.2 & 31 \\
\hline 13 & PBA & 120 & 4.1 & 22 & 85 & $30(75)^{\mathrm{c}} \quad 80^{\mathrm{d}}$ & $8.2^{\mathrm{c}} \quad 0.14^{\mathrm{d}}$ & $1.3^{\mathrm{c}} 1.34^{\mathrm{d}}$ & 2.2 & 36 \\
\hline 14 & PBA & 60 & 5.2 & 22 & 98 & $68(95)^{\mathrm{c}} 530^{\mathrm{d}}$ & $1.0^{\mathrm{c}} 110^{\mathrm{b}, \mathrm{d}}$ & $1.5^{\mathrm{c}} 10^{\mathrm{d}}$ & 2.0 & 33 \\
\hline 15 & PVP & 1.0 & 4.7 & 22 & 85 & $30(74)^{\mathrm{c}} \quad 80^{\mathrm{d}}$ & $23^{\mathrm{c}} \quad 0.15^{\mathrm{d}}$ & $1.3^{\mathrm{c}} \quad 1.3^{\mathrm{d}}$ & & \\
\hline
\end{tabular}

${ }^{\mathrm{a}}$ Recipe $c f$. Exptl. part., [BA] $=1.56 \mathrm{~mol} \mathrm{dm}{ }^{3}$, [APS] $=1.0 \times 10^{-3} \mathrm{~mol} \mathrm{dm}^{-3}, \quad{ }^{\mathrm{b}} N_{\mathrm{p}} \times 10^{-12}\left(\mathrm{dm}^{-3}\right)$. ${ }^{\mathrm{c}}$ Fraction of small latex particles. ${ }^{\mathrm{d}}$ Fraction of large latex particles.

Table III. Kinetics, molecular weights and colloidal parameters in the radical polymerization of BA in fine emulsions ${ }^{\text {a }}$

\begin{tabular}{|c|c|c|c|c|c|c|c|c|c|}
\hline \multirow{2}{*}{ Run } & \multirow{2}{*}{$\frac{[\mathrm{APS}] 10^{3}}{\mathrm{~mol} \mathrm{dm}^{-3}}$} & \multirow{2}{*}{$\frac{\mathrm{R}_{p, \max } \times 10^{3}}{\operatorname{mol~dm}{ }^{-3} \mathrm{~s}^{-1}}$} & \multirow{2}{*}{$\frac{\text { Conv }_{\text {max }}}{\%}$} & \multirow{2}{*}{$\frac{\text { Conv.f }_{\text {f }}}{\%}$} & \multirow{2}{*}{$\frac{D}{\mathrm{~nm}}$} & \multirow{2}{*}{$\frac{N_{\mathrm{p}} \times 10^{-18}}{\mathrm{dm}^{-3}}$} & \multirow{2}{*}{ APSD } & \multirow{2}{*}{$M_{\mathrm{v}} \times 10^{6}$} & \multirow{2}{*}{$\frac{\gamma}{\mathrm{mN} \mathrm{cm}}$} \\
\hline & & & & & & & & & \\
\hline 16 & $0.63^{\mathrm{b}}$ & 2.3 & 35 & 94 & $52^{\mathrm{k}}$ & $1.16^{\mathrm{k}}$ & $1.1^{\mathrm{k}}$ & 1.6 & 40 \\
\hline 17 & $3.2^{\mathrm{b}}$ & 4.5 & 30 & 94 & $51^{\mathrm{k}}$ & $1.2^{\mathrm{k}}$ & $1.1^{\mathrm{k}}$ & 1.4 & 37 \\
\hline 18 & $6.3^{\mathrm{a}}$ & 5.4 & 28 & 96 & $51^{\mathrm{k}}$ & $1.2^{\mathrm{k}}$ & $1.1^{\mathrm{k}}$ & 1.4 & 35 \\
\hline 19 & $0.63^{\mathrm{c}}$ & 1.8 & 19 & 78 & $51^{\mathrm{k}}$ & $1.1^{\mathrm{k}}$ & $1.5^{\mathrm{k}}$ & 1.4 & 40 \\
\hline 20 & $3.2^{\mathrm{c}}$ & 4.0 & 25 & 82 & $53^{\mathrm{k}}$ & $0.95^{\mathrm{k}}$ & $1.5^{\mathrm{k}}$ & 1.3 & 36 \\
\hline 21 & $6.3^{\mathrm{c}}$ & 5.0 & 35 & 87 & $55^{\mathrm{k}}$ & $0.91^{\mathrm{k}}$ & $1.4^{\mathrm{k}}$ & 1.2 & 33 \\
\hline 22 & $0.63^{\mathrm{d}}$ & 1.7 & 20 & 86 & $51(95)^{\mathrm{k}} 260^{\mathrm{l}}$ & $1.1^{\mathrm{k}} 4.3^{\mathrm{j}}$ & $1.43^{\mathrm{k}}$ & 1.3 & 32 \\
\hline 23 & $0.63^{\mathrm{e}}$ & 4.2 & 22 & 84 & $41(86)^{\mathrm{k}} \quad 94^{\mathrm{l}}$ & $1.8^{\mathrm{k}} 0.025^{\mathrm{l}}$ & $1.45^{\mathrm{k}} 1.4^{\mathrm{i}}$ & 2.0 & 33 \\
\hline 24 & $0.63^{\mathrm{f}}$ & 5.2 & 25 & 92 & $60^{\mathrm{k}}$ & $0.65^{\mathrm{k}}$ & $1.35^{\mathrm{k}}$ & 2.0 & 33 \\
\hline 25 & $0.63^{\mathrm{g}}$ & 4.2 & 21 & 92 & $68(95)^{\mathrm{k}} 530^{\mathrm{l}}$ & $0.5^{\mathrm{k}} 0.5^{\mathrm{j}}$ & $1.5^{\mathrm{k}} 10^{\mathrm{i}}$ & 1.9 & 33 \\
\hline 26 & $0.63^{\mathrm{h}}$ & 4.1 & 22 & 85 & $30(74)^{\mathrm{k}} \quad 80^{\mathrm{l}}$ & $4.0^{\mathrm{k}} 0.075^{\mathrm{l}}$ & $1.3^{\mathrm{k}} \quad 1.3^{\mathrm{i}}$ & 2.0 & 36 \\
\hline 27 & $0.63^{\mathrm{i}}$ & 3.7 & 21 & 92 & 62 & $0.67^{\mathrm{k}}$ & $1.6^{\mathrm{k}}$ & 1.7 & 32 \\
\hline
\end{tabular}

${ }^{\mathrm{a}}$ Recipe $c f$. Exptl. part. $[\mathrm{BA}]=0.78 \mathrm{~mol} \mathrm{dm}^{-3} .{ }^{\mathrm{b}}$ Without polymer. ${ }^{\mathrm{c}}$ With $0.2 \mathrm{~g}$ PSt $\left(M_{w}=1.8 \times 10^{6}\right) / 100 \mathrm{~g}$ water. ${ }^{\mathrm{d}} \mathrm{PSt}\left(M_{w}=9.3 \times 10^{4}\right)$. ${ }^{\mathrm{e}} \mathrm{PMMA}\left(M_{w}=1.9 \times 10^{4}\right)$. ${ }_{\mathrm{f}} \mathrm{PMMA}\left(M_{w}=1.6 \times 10^{5}\right) .{ }_{\mathrm{g}} \mathrm{PBA}\left(M_{w}=6.1 \times 10^{4}\right) .{ }^{\mathrm{h}} \mathrm{PBA}\left(M_{w}=1.2 \times 10^{6}\right) .{ }^{\mathrm{i}} \mathrm{PVP}\left(M_{w}=1.0 \times 10^{4}\right) .{ }^{\mathrm{j}} \mathrm{Np} \times 10^{-14}$ $\left(\mathrm{dm}^{-3}\right) .{ }^{\mathrm{k}}$ Fraction of small latex particles. ${ }^{\mathrm{l}}$ Fraction of large latex particles.

cleated particles and amount of micellar emulsifier.

It is interesting to note that the surface tension $((\gamma)$ decreases with increasing [APS] and $\gamma$ is lower for the runs with PSt (Tables I and II). $\gamma$ is expected to increase with particle surface area due to decrease of free emulsifier fraction. The results show that the reverse is true. This might be attributed to formation and accumulation of surface active oligomers in the continuous phase. ${ }^{26}$ The decrease in $\gamma$ is more pronounced in runs with predissolved PSt (total interface area strongly increased). Incompatibily between PSt and (undissociated) emulsifier is expected to decrease the amount of emulsifier dissolved in the droplet core (or increase micellar fraction of emulsifier).

\section{Colloidal Parameters}

The addition of polymeric hydrophobe (PSt, PMMA, PBA, and PVP) promotes the formation of polymer latexes with bimodal size distribution (Tables I and II). Similar behavior was reported by $\mathrm{P}$. J. Blythe et al. ${ }^{15}$ and Reimers and Schork. ${ }^{11}$ This behavior is less distinct in systems with larger weight ratio emulsifier/monomer (Table III). The high molecular weight PSt did not promote the formation of latex particles with bimodal particle size distribution (PSD) but average particles size slightly increased (runs 19-21). Similar behavior was found with PVP. The latex particles with bimodal PSD and fraction of large particles appear with the medium molecular weight PSt and PBA $\left(c a .1 \times 10^{5}\right)$. The bimodal PSD with smaller particles appears with PMMA $\left(M_{w}=\right.$ $\left.1.9 \times 10^{4}\right)$ and PBA $\left(1 \times 10^{6}\right)$.

Final particle size $(D)$ and $N_{\mathrm{p}}$ were found to vary with increasing [APS] as follows (Table I):

$D \propto[\mathrm{APS}]^{-0.11}$ and $N_{\mathrm{p}} \propto[\mathrm{APS}]^{0.4}$ (runs $1-4$, the system A)

$D_{\mathrm{s}} \propto$ [APS $^{-0.46}, D_{1} \propto$ [APS $^{-0.92}, N_{\mathrm{ps}} \propto[\mathrm{APS}]^{1.23}$ and $N_{\text {pl }} \propto[\text { APS }]^{4.1}$ (runs 5-8, the system B)

where the subscripts $\mathrm{s}$ and 1 denote small and large particles. In both runs, $D$ decreases (or $N_{\mathrm{p}}$ increases) with increasing APS concentration and the decrease is more pronounced with seed polymer. The reaction order $y\left(N_{\mathrm{p}}\right.$ $\propto[\text { APS }]^{y}$ ) for the system $\mathrm{A}$ is in good agreement with the micellar model $(y=0.4)$. In system $\mathrm{B}, N_{\mathrm{p}}$ strongly increases with APS concentration; i.e., the reaction order $y$ is $\mathbf{1 . 2 3}$ for small particles and $\mathbf{4 . 1}$ for large particles. The presence of polymer decreases monomer droplet degradation and preserves microdroplet for a longer reaction time. Thus, the predominant cause of "enhanced nucleation" from the addition of polymer is that the polymer adds extra stability to monomer droplets both prior to and during polymerization. The enhancement is primary attributed to preservation of the droplet number by the presence of polymer in each monomer droplet. ${ }^{15,27}$

The slightly weaker dependence of $N_{\mathrm{p}}$ on [initiator] was reported by Delgado et al. ${ }^{2}$ for miniemulsion copolymerization of vinyl acetate and butyl acrylate. For 
macroemulsions, the order was found to be 0.0 , and for miniemulsions, 0.8. Fontenot and Schork ${ }^{28}$ reported the reaction order $y=0.11$ for miniemulsion and 0.28 for the macroemulsion polymerization of MMA. The data presented for miniemulsion indicated a leveling of particle number at high initiator concentration. This is believed to correspond to the point at which all monomer droplets become nucleated. The reverse is true for current systems where the dependence $N_{\mathrm{p}} v s$. [APS] (a log-log plot) has nearly exponential trend (Table I). This behavior is connected with increased number of monomer droplets or radical entry efficiency. In the former case, the release of emulsifier from the monomer phase may increase the number of micelles. ${ }^{17}$ In the latter case, the accumulated polymer might modify the droplet surface for successful radical entry.

The presence of a large amount emulsifier as well as coemulsifier $(\mathrm{BA})^{9,17}$ assures the formation of very small monodisperse microdroplets and polymer particles. ${ }^{1} \mathrm{Ta}-$ bles I and III show that the microemulsion polymerization of BA forms relatively monodisperse polymer particles. The addition of polymer leads to the formation of unique polymer latexes with bimodal APSD. The presence of large particles is connected with the monomer emulsification and mixed mode of particle nucleation. In the microemulsion polymerization of styrene or BA, the initial abrupt increase in turbidity (or particle size) was attributed to the formation of polymer. ${ }^{1,3}$ The fraction of nucleated particles, however, is very small compared to the fraction of thermodynamically stable microdroplets as well as its effect on the whole polymerization process and colloidal parameters. This may not be true for current systems where each microdroplet contains one or more polymer chains.

APSD is nearly independent of [APS] with and without polymeric hydrophobe. APSD is much broader in runs with hydrophobe. APSD of large (l) particles is much larger than that of small (s) ones.

\section{Molecular Weight Parameters}

Variation of the viscosity-average molecular weights with initiator concentration and reaction conditions is summarized in Tables I-III. The molecular weight decreases with increasing [APS] in the following order :

$M_{\mathrm{v}} \propto[\mathrm{APS}]^{-0.1}($ runs $1-4), M_{\mathrm{v}} \propto[\mathrm{APS}]^{-0.1}($ runs $5-$ 8) (Table I)

$M_{\mathrm{v}} \propto[\mathrm{APS}]^{-0.06}($ runs $16-18), M_{\mathrm{v}} \propto[\mathrm{APS}]^{-0.06}$ (runs 19-21) (Table III)

The data show a slight dependence of $M_{\mathrm{v}}$ on the APS concentration, attributed to the governing role of the chain transfer to monomer and/or emulsifier, desorption of transferred radical from the particles into the continuous phase and re-entry of transferred radicals. The decrease in $M_{\mathrm{v}}$ is more pronounced for the higher weight ratio BA/SDS. $M_{\mathrm{v}}$ is somewhat lower in runs with polymeric hydrophobe PSt, due to decreased degradation of monomer droplets and lower monomer concentration at the reaction loci. A similar trend for both systems results from the dominant contribution of small particles (with or without polymer). A similar trend in $M_{\mathrm{v}}$ is evident from PMMA and PBA (Table II). Smaller polymer is also formed with PVP.

Table III shows that the lower weight ratio BA/SDS favors the formation of lower molecular weight polymers. This is attributed to the formation of small microdroplets with a less distinct monomer core; that is, monomer is more diluted with emulsifier. Under these circumstances, the chain-transfer to emulsifier is significant. The chain-transfer to emulsifier decreases polymerization rate and molecular weight. ${ }^{15,24}$ The dilution of the monomer phase by hydrophobic alkyl chains of SDS increases the chain-transfer to emulsifier. ${ }^{20,29}$ However, the relatively large polymers were generated in runs 23 -26 with PMMA and PBA. This may result from the location of polar polymer at the droplet surface leading to different roles of dissolved polymer.

\section{CONCLUSION}

Polymerization rate (with polymer) vs. conversion curve shows nonconstant rate intervals. The presence of the first rate maximum is the result of increase of particle number and decrease of monomer concentration at the reaction loci. The addition of polymeric hydrophobe increases the maximum rate of polymerization as a result of formation of a larger number of particles. The rate shoulder at ca. 50-70\% conversion and polymer particles with bimodal distribution appear in the runs with seed polymer. The maximum rate of polymerization increases with initiator concentration and the increase is more pronounced with predissolved polymer. The enhanced rate of polymerization and particle formation with predissolved polymer results from the preservation of monomer droplets during polymerization. The continuous particle nucleation results from the large number of free monomer-swollen micelles and release of emulsifier from the nucleated particles. The decrease in polymerization rate with increasing conversion is attributed to decrease of monomer concentration at reaction loci and increased chain-transfer to emulsifier.

Acknowledgment. This research was supported by the Slovak Grant Agency (VEGA) through grant numbers 2/5005/98. The author thanks Mrs. O. Jurikova for assistance in the experiments.

\section{REFERENCES}

1. J. S. Guo, E. D. Sudol, J. W. Vanderhoff, and M. S. Elaasser, J. Polym. Sci., Part A. Polym. Chem., 30, 691 (1992); ibid. 30, 703 (1992).

2. J. Delgado, M. S. El-Aasser, C.A.Sileb, and J. W. Vanderhoff, J. Polym. Sci., Polym.Chem., Ed., 28, 777 (1990).

3. I. Capek, Adv. Colloid Interface Sci., 80, 85 (1999).

4. P. Potisk and I. Capek, Angew. Makromol. Chem., 222, 125 (1994).

5. Y. T. Choi, M. S. El-Aasser, E. D. Sudol, and J. W. Vanderhof, J. Polym. Sci., Polym. Chem. Ed., 23, 2973 (1985).

6. J. Ugelstad, M. S. El-Aasser, and J. W. Vanderhoff, J. Polym. Sci., Polym. Lett. Ed, 111, 503 (1973).

7. I. Capek, V. Juranicova, K. Ito, J. Barton, and J. M. Asua, Polym. Int., 43, 1 (1997).

8. T. Tanrisever, O. Okay, and I. C. Sonmezoglu, J. Appl . Polym. Sci., 61, 485 (1996).

9. I. Capek and P. Potisk, Eur. Polym. J., 31, 1269 (1995).

10. I. Capek and W. Funke, Makromol. Chem,, 191, 2549 (1990).

11. J. L. Reimers and F. J. Schork, J. Appl. Polym. Sci., 60, 251 (1996).

12. R. Hunter, "Foundation in Colloid Science", Oxford Press, 
New York, N.Y., 1989.

13. I. Capek and J. Barton, Makromol. Chem., 186, 1297 (1985).

14. S. H. Maron, M. E. Elder, and I. N. Ulevitch, J. Colloid Sci., 9, 89 (1954)

15. P. J. Blythe, A. Klein, E. D. Sudol, and M. S. El-Aasser, Macromolecules, 32, 4225, 6944, 6952, (1999).

16. I. Capek and C. S. Chern, Adv. Polym. Sci., in press.

17. S. Y. Lin, C. S. Chern, and I. Capek, Polym. J., in press.

18. N. Garti and A. Aserin, Adv. Colloid Interface Sci., 65, 37 (1996).

19. N. Muller, J. Colloid Interface Sci., 63, 383 (1978).

20. M. Nomura and K. Suzuki, Makromol. Chem. Phys., 198, 3025 (1997).

21. M. G. Zhang, Z. X. Weng, Z. M. Huang and Z. R. Pan, Eur. Polym. J., 34, 1243 (1998).
22. J. Barton, V. Juranicova, and V. Vaskova, Makromol. Chem., 186, 1935 (1985); ibid., 1861943 (1985).

23. P. L. Tang, E. D. Sudol, C. A. Silebi, and M. S. El-Aasser, J. Appl. Polym. Sci., 43, 1059 (1991).

24. C. M. Miller, E. D. Sudol, C. A. Silebi, and M. S. El-Aasser, Macromolecules, 28, 2765 (1995).

25. W. V. Smith and R. H. Ewart, J. Am. Chem. Soc., 70, 3695 (1948).

26. K. Tauer, R. Deckwer, I. Kuhn, and C. Schellenberg, Colloid Polym. Sci., 277, 607 (1999).

27. K. Landfester, N. Bechthold, F. Tiarks, and M. Antonietti, Macromolecules, 32, 2679 (1999).

28. K. Fontenot and F. J. Schork, J. Appl. Polym. Sci., 49, 633 (1993).

29. I. Capek and P. Potisk, J. Polym. Sci., Polym. Chem., 33, 1675 (1995). 